\title{
Percepciones sobre la vacunación de la rabia silvestre en población Awajún de la provincia de Condorcanqui, Amazonas, Perú, 2013
}

I 1 Gloria Carmona Clavijo, 2 Jose Daza Arevalo,

${ }^{3}$ Víctor Luis Osorio Pretel, ${ }^{4}$ Julio Portocarrero Gutiérrez I

Resumen: El objetivo de estudio fue conocer las percepciones sobre la vacunación contra la rabia silvestre en comunidades nativas de la etnia Awajún, en Amazonas, Perú, 2012. Realizamos un estudio cualitativo a través de entrevistas de baja estructuración, para recoger las percepciones de la población, autoridades locales y personal de salud. Las percepciones sobre las causas de la RS fueron atribuidas a murciélagos portadores de alguna infección, veneno y daño sobrenatural, o al efecto de brujería. Manifestaron el efecto mortal de la RS e información variada sobre la posibilidad o ausencia de tratamiento. Como medidas de prevención refirieron el uso de mosquiteros, mallas, tramperas, infusiones e ungüentos, luego mencionaron a la vacuna. Sobre la vacunación contra la RS, refirieron haber recibido información del personal de salud, manifestando argumentos para oponerse o cuestionarla como: el objetivo encubierto de perjudicar el desarrollo del pueblo Awajún, no ser una vacuna necesaria y la generación de efectos negativos en niños y adultos. Las percepciones recogidas hacia este tipo de vacunación manifiestan diversos cuestionamientos sobre su potencial beneficio o necesidad en la comunidad y están relacionadas tanto a aspectos religiosa-culturales y socioeconómicos como a las relaciones de comunicación con el estado y el sistema de salud peruano.

> Palabras-clave: vacunas antirrábicas; rabia; población indígena; percepción social

\author{
${ }^{1}$ Centro Nacional de Salud \\ Pública, Instituto Nacional \\ de Salud, Lima, Perú \\ (gloriacarmona.c@gmail.com). \\ ${ }^{2}$ Dirección Regional de Salud. \\ Amazonas, Condorcanqui, Perú \\ (jlda38@hotmail.com). \\ ${ }^{3}$ Red de Salud de \\ Condorcanqui. Amazonas, \\ Condorcanqui, Perú \\ (osoriocondor@hotmail.com). \\ ${ }^{4}$ Instituto Nacional de Salud, \\ Centro Nacional de Salud \\ Intercultural. Lima, Chorrillos, \\ Perú (juliocesarportocarrero@ \\ hotmail.com).
}

Recibido: 03/03/2015 Aprobado: 10/08/2015 


\section{Introducción}

El número de muertes por el virus de la rabia humana a nivel mundial, para el año 2010, se estima en 61,000 muertes, la mayoría (84\%) ocurre en zonas rurales, y afecta principalmente a niños de África, China e India y es rabia urbana transmitida por la mordedura de perros infectados WHO, 2013). La rabia silvestre (RS) es un problema de salud pública en las zonas subtropicales y tropicales de América, desde México hasta Argentina. El principal reservorio es el murciélago hematófago Desmodus rotundus, que transmite la RS a los animales domésticos y a seres humanos, principalmente en la amazonia (SCHNEIDER et al., 2009).

En el Perú, a partir de 1975 se comienza a conocer la implicancia de la RS en la salud pública, al reportarse en el departamento de Amazonas la muerte de 13 personas nativas con el antecedente de mordeduras frecuentes de murciélagos (NAVARRO, 2001; ZOONOSIS, 2008). Desde 1975 al 2010 se notificaron más de 250 muertes por RS, la mayoría ocurrieron en las comunidades amazónicas nativas awajunas de Condorcanqui (CANAP) y en menores de 15 años (MINISTERIO DE SALUD, 2011a). En este contexto, la Organización Mundial de la Salud (OMS) recomienda la aplicación de la vacunación pre exposición (VAPEX) en zonas de transmisión de RS, por su efectividad en lograr niveles de anticuerpos protectores (GÓMEZ, 2011; WHO, 2011; WHO, 2007).

La etnia Awajún representa el segundo grupo indígena más numeroso de la amazonia peruana después de los Ashaninka (BDPI, 2014). El riesgo hacia la RS en las CANAP es causado por distintos factores como; las características de sus viviendas, su habitad trópico rural y la cotidianidad con los murciélagos, además de sus particulares concepciones del proceso de salud-enfermedad, características socio-económicas y la posibilidad de adopción de medidas preventivas (SCHNEIDER et al., 2009).

En respuesta a este riesgo, el Ministerio de Salud del Perú planteó a la Dirección Regional de Salud Amazonas la necesidad de implementar la VAPEX en las CANAP; es así que en julio del 2011, se inició la campaña de vacunación en la provincia de Condorcanqui, con el objetivo de proteger contra la RS a las CANAP que habitan estas áreas geográficas. Sin embargo, la efectividad 
de las intervenciones en salud pública está relacionada no solamente con su diseño teórico, sino también con su contextualización. Es complejo garantizar que las normas definidas para mejorar la salud pública terminen siendo beneficiosas cuando las actividades que las integran se ven condicionadas por las concepciones de la comunidad objetivo (RYCHETNIK et al., 2002). En alusión a ello, la política de salud establece que el desarrollo de todo tipo de intervención comunitaria debe adaptarse a una pertinencia socio-cultural; respetando la identidad, disminuyendo la desigualdad, promoviendo la equidad y manteniendo un enfoque basado en género (GOMEZ, 2004). En este proceso sólo una comunicación articulada y reflexiva que considere tanto los aspectos socio-económicos-culturales como los aspectos de la facilidad y accesibilidad a los servicios de salud puede viabilizar la efectividad del programa de vacunación y de sensibilización hacia la RS.

Así, aspectos que tienen que ver con la aceptabilidad a la VAPEX deben ser tomados en cuenta, ya que pueden incrementar la brecha de acceso a esta vacuna. Por tal, el objetivo del presente estudio buscó describir las percepciones en relación a la VAPEX contra la RS en la población Awajún de seis CANAP de la provincia de Condorcanqui.

\section{Materiales y Métodos}

\section{Diseño del estudio}

Realizamos un estudio cualitativo que utiliza un enfoque de Procedimiento de Asesoría Rápida (RAP) para la investigación social en salud. El RAP tiene como objetivo aplicar herramientas etnográficas adaptadas para un trabajo de campo de corto plazo en las comunidades seleccionadas para el estudio (SCRIMSHAW; HURTADO, 1987). Trabajamos con técnicas de recojo de datos de baja estructuración destinadas a recoger el punto de vista y vocabulario de la población de estudio (SCHENSUL, 1999).

\section{Descripción de la población de estudio}

Las seis CANAP de la etnia Awajún participantes en este estudio (Urakusa, Seasme, Shajain. Kayants, Pajacusa y Ajachin), están conformadas desde 200 
hasta 450 habitantes en cada una, ubicadas en la provincia de Condorcanqui, a más de tres horas de la ciudad de Nieva a través de embarcaciones fluviales. Todas se encuentran dentro de la jurisdicción del (C.S) de Uracusa, cuyo acceso dependía, en la mayoría de los casos, del transporte fluvial. Hasta inicios del siglo $\mathrm{XX}$, su principal fuente de sustento era el bosque tropical, de donde obtenían los recursos necesarios para su reproducción física y cultural, a través de actividades como la horticultura, la cacería, la recolección y la pesca. Como muchas otras sociedades amazónicas, actualmente los awajún están experimentando un rápido proceso de cambio cultural, económico y ecológico (FULLER, 2009). Los cambios señalados afectaron los patrones de asentamiento, organización política, educación, cosmovisión y la socialización de este pueblo (MAINVILLE, 2014). Actualmente muchos de sus asentamientos están integrados al sistema de carreteras, lo cual ha modificado de manera significativa sus patrones productivos y los sistemas de aprovechamiento de los recursos naturales. Por otro lado, existe también un intenso flujo de migrantes provenientes de la sierra y otras regiones. Todas estas migraciones han generado un gradual proceso de mestizaje (BROWN, 1984). No obstante, poco se sabe sobre los efectos de estos cambios sobre la salud y las prácticas tradicionales de esta población.

En relación a sus condiciones de vida; la agricultura, la artesanía, los criaderos de animales, y la carpintería son sus principales actividades laborales, sin contar aún con servicios de saneamiento básico; utilizando silos y letrinas comunales. La religión presenta gran influencia a través de sus líderes o pastores religiosos. La situación de salud de estas CANAP es imprecisa a causa de factores como el sub-registro, el limitado acceso geográfico, omisión de identificación nacional o certificación incompleta e insuficiente personal de salud para la atención de áreas rurales extensas lo que dificulta la atención de las demandas de salud y del diálogo con la comunidad. Estas comunidades tienen dificultades en la accesibilidad al C.S tanto por el transporte terrestre o fluvial requerido como por los costos que estos traslados implican, lo cual se dificulta por su condición de pobreza extrema, existiendo familias que no llegan a acudir al C.S ni a ser visitadas por estos factores. 
Realizamos entrevistas grabadas, abiertas y semiestructuradas; observación participante de baja estructuración y revisión de documentos. Durante la observación participante, se acompañó a varias campañas de vacunación realizadas en la zona de estudio para escuchar los discursos y observar las reacciones de la población, así como las características de las viviendas y condiciones de vida que favorecen la exposición a la mordedura por murciélagos, los cuales fueron registrados en diarios de campo, lo mismos que permitieron la identificación de ítems emergentes que podrían ser conversados durante las entrevistas y la adaptación necesaria al contexto local.

Las entrevistas fueron dirigidas a pobladores y autoridades de las comunidades estudiadas, hasta lograr la saturación de información. Las entrevistas fueron realizadas por el investigador principal en las viviendas de los pobladores y centros de salud, con una duración de veinticinco minutos como promedio. Durante las entrevistas a autoridades locales y pobladores, se contó con el acompañamiento permanente de intérpretes locales. Se utilizaron preguntas indirectas, a fin de evitar la impresión de confrontación o de criterio preguntándose sobre las opiniones o perspectivas del participante o de sus pares, en lugar de preguntar directamente sobre las decisiones o experiencias personales (BERNARD, 2012).

\section{Estrategia de selección de casos y muestreo}

Se seleccionaron por conveniencia seis comunidades con riesgo de transmisión de RS y con tasa de mordeduras por murciélagos en población general mayor del 2\% (MINISTERIO DE SALUD, 2011b). Al interior de cada comunidad se trabajó con un muestreo por cuotas, a fin de que distintos actores comunitarios compartan desde información variada y adicional sobre el mismo tema aspirando así, al principio de variación máxima (MILES; HUBERMAN, 1994). Estos actores fueron los líderes comunitarios, pastores religiosos y docentes de cada comunidad. A su vez estos líderes fueron identificados durante las entrevistas realizadas a los residentes e invitados a participar voluntariamente del estudio (ver tablas 1 y 1.1). 
Tabla 1. Características de los entrevistados según actor social, sexo, idioma y edad

$$
\text { Sexo }
$$

Edad

Entrevista. Idioma

$\mathrm{N}=32$

Varón: Mujer:

$\mathrm{n}=20 \quad \mathrm{n}=12 \quad$ Min $\quad$ Max $\quad$ Med Awajun 8 Español 21 Ambos 3

Autoridades Comunales

$\begin{array}{llllllllll}\text { Apu } & 6 & 6 & 0 & 27 & 48 & 37.6 & 0 & 5 & 1 \\ \text { Docentes } & 4 & 3 & 1 & 29 & 58 & 47.8 & 0 & 4 & 0 \\ \text { Pastores } & 4 & 4 & 0 & 35 & 48 & 41.3 & 0 & 3 & 1\end{array}$

Comuneros

$\begin{array}{llllllllll}\text { Urakusa } & 3 & 2 & 1 & 35 & 58 & - & 1 & 1 & 1 \\ \text { Ajachin } & 3 & 2 & 1 & 24 & 44 & - & 1 & 2 & 0 \\ \text { Kayants } & 3 & 1 & 2 & 18 & 44 & - & 2 & 1 & 0 \\ \text { Seasme } & 3 & 1 & 2 & 41 & 49 & - & 2 & 1 & 0 \\ \text { Shajain } & 3 & 1 & 2 & 40 & 54 & - & 0 & 3 & 0 \\ \text { Pajacusa } & 3 & 1 & 2 & 29 & 55 & - & 2 & 1 & 0\end{array}$

Fuente: elaboración propia

Tabla 1.1. Características de los entrevistados según ocupación y nivel de educación por sexo

\begin{tabular}{|l|l|c|c|}
\hline \multicolumn{2}{|l|}{ Sexo } & Varon: 20 & Mujer: $\mathbf{1 2}$ \\
\hline \multirow{4}{*}{ Ocupación } & Agricultor & 15 & 4 \\
\cline { 2 - 4 } & Profesor & 5 & 1 \\
\cline { 2 - 4 } & Artesana & 0 & 2 \\
\cline { 2 - 4 } & Ama de casa & 0 & 5 \\
\hline \multirow{5}{*}{ Educación } & Superior & 2 & 0 \\
\cline { 2 - 4 } & Secundaria completa & 8 & 2 \\
\cline { 2 - 4 } & Secundaria incompleta & 6 & 4 \\
\cline { 2 - 4 } & Primaria incompleta & 2 & 4 \\
\cline { 2 - 4 } & Primaria completa & 2 & 2 \\
\cline { 2 - 4 } & Analfabeta & 0 & 0 \\
\hline
\end{tabular}

Fuente: elaboración propia 
Los criterios de diseño, descripción y análisis del presente estudio están sujetos al COREQ Checklist (TONG et al., 2007). Las entrevistas y las anotaciones adicionales fueron transcritas en un procesador de textos y codificadas a través del software para análisis cualitativo ATLAS, ti (versión de prueba). El análisis se dividió en las siguientes fases: (1) elaboración de notas descriptivas y memos producto de la recolección de los datos, (2) lectura de las entrevistas y elección de códigos, (3) nueva lectura de entrevistas y codificación, (4) análisis por códigos, diferenciando entre comunidades y tipo de actor, y (5) elaboración de matrices de concentración de datos según códigos a partir de la información encontrada en las entrevistas, permitiendo focalizar resultados y establecer una relación con la teoría existente.

\section{Aspectos éticos}

El estudio fue conducido dentro de los patrones éticos exigidos por la Declaración de Helsinki y aprobado por el Comité Institucional de Ética del Instituto Nacional de Salud de Perú. Se obtuvo el consentimiento libre e informado de las autoridades regionales y locales para acceder a las comunidades seleccionadas, garantizándoles la confidencialidad e anonimato de la información compartida. Este proceso concluyo con la firma de cada uno de los entrevistados. La socialización, difusión y discusión de los resultados de este estudio se realizaron en el 2014 realizándose con autoridades regionales y con las autoridades locales; en las que participaron los líderes comunitarios, personal de salud local, sociedad civil, autoridades municipales y de educación.

\section{Hallazgos}

Se realizó un total de 32 entrevistas directas incluyendo a Apus (autoridades locales tradicionales), docentes, autoridades religiosas y comuneros. En una primera sección se describen las percepciones de la población sobre la enfermedad de RS, identificando puntos en común y diferencias entre comunidades. En la segunda sección se describen y analizan las percepciones de la población sobre la vacunación contra la RS. 


\section{Perspectivas sobre la RS}

Los entrevistados mencionaron distintas causas atribuidas a la RS y la existencia de algún tipo de cura, relacionadas a sus percepciones culturales y socio-económicas del proceso de salud-enfermedad, así como su cotidianidad con las mordeduras de murciélago, alternativas de prevención y abordaje (tabla 2). Expresaron también las barreras socio-económicas que encuentran en la ejecución de las medidas preventivas referidas.

Tabla 2. Principales percepciones sobre la rabia silvestre, según comunidades

\begin{tabular}{|c|c|c|c|c|c|c|}
\hline \multirow{2}{*}{$\begin{array}{l}\text { Percepciones } \\
\text { sobre la RS }\end{array}$} & \multicolumn{6}{|c|}{ Comunidades nativas awajunas. Condorcanqui, Amazonas } \\
\hline & Comunidad 1 & Comunidad 2 & Comunidad 3 & Comunidad 4 & Comunidad 5 & Comunidad 6 \\
\hline Causas & $\begin{array}{l}\text { Murciélago } \\
\text { infectado }\end{array}$ & Murciélago & $\begin{array}{l}\text { Causa } \\
\text { incomprendida, } \\
\text { ya que no existía } \\
\text { antes. Han } \\
\text { escuchado que es } \\
\text { por murciélagos }\end{array}$ & $\begin{array}{l}\text { Murciélago y } \\
\text { daño espiritual }\end{array}$ & $\begin{array}{l}\text { Algunos } \\
\text { murciélagos } \\
\text { dominados por } \\
\text { el diablo. }\end{array}$ & $\begin{array}{l}\text { No existe en el } \\
\text { hombre, sólo } \\
\text { en animales }\end{array}$ \\
\hline Tratamiento & Si tiene cura & \multicolumn{3}{|l|}{ No tiene cura } & Si tiene cura & no existe \\
\hline $\begin{array}{l}\text { Percepción del } \\
\text { daño }\end{array}$ & $\begin{array}{l}\text { Puede } \\
\text { ocasionar } \\
\text { muerte }\end{array}$ & Muerte & Muerte por brujer & ía, por daño espiri & tual. & $\begin{array}{l}\text { La mordedura } \\
\text { de murciélago } \\
\text { es normal sin } \\
\text { originar mayor } \\
\text { daño. }\end{array}$ \\
\hline $\begin{array}{l}\text { Principales } \\
\text { zonas de } \\
\text { mordedura }\end{array}$ & Cabeza, pies & & & Cabeza. & Pies & $\begin{array}{l}\text { Cabeza, pies, } \\
\text { orejas }\end{array}$ \\
\hline $\begin{array}{l}\text { Principal sujeto } \\
\text { mordido }\end{array}$ & Niños & & & & & \\
\hline
\end{tabular}

Fuente: elaboración propia

\section{Causas atribuidas}

Si bien se identificaron diferentes percepciones en torno a la causalidad de la RS, dos son las principales. En primer lugar, se encontró un grupo de pobladores que consideraba que la rabia era una enfermedad propia de algunos animales (como los perros y murciélagos) y no de seres humanos. Según estos informantes, los casos humanos atribuidos a la RS son síndromes de origen sobre natural, principalmente daño o brujería causada por otras personas. Dentro de este grupo 
algunos consideraban que el murciélago podría ser un transmisor del daño o brujería y no de una enfermedad de origen natural.

El murciélago me pica pero no me da rabia, porque saldría rabia? Acaso es un perro, un animal? La persona nunca tiene rabia entonces no puedes morir por rabia. Rabia tiene los animales, perro, chancho, esos tienen. (Apu comunitario).

En segundo lugar, una mayoría de nuestros informantes consideró que la RS era una enfermedad generada por la mordedura de murciélagos. Sin embargo el agente causante de la enfermedad difería de un testimonio a otro. Así, mientras que para algunos el murciélago era portador de un veneno, para otros estaba infectado con la enfermedad. Otro grupo, indicó no entender cómo es que los murciélagos, con los que conviven cotidianamente compartiendo su hábitat y por el acceso permanente que tienen a sus casas por el precario material de construcción, podía originar RS, considerando que muchos de ellos tienen mordeduras pero no han desarrollado la enfermedad y que, desde su punto de vista, sus antepasados no la han padecido. Se debe señalar que un mismo informante podía plantear varias explicaciones dentro de un mismo discurso.

\section{Efectos y tratamiento}

La gran mayoría de los entrevistados considera la RS una enfermedad mortal e indica que desconocen de la existencia de un tratamiento o cura. Sin embargo en dos de seis comunidades estudiadas algunos informantes indicaron el uso de "medicinas" o "ampollas" como tratamiento aunque no pudieron especificar el tipo de medicación a utilizar. Se debe señalar que en estas dos comunidades algunos informantes señalaron que las vacunas eran un tratamiento de RS.

\section{Prevención}

La mayoría de los entrevistados consideró que la principal forma de prevención de la RS consistía en evitar la mordedura de murciélago que ataca principalmente a los menores mientras duermen, especialmente en la zona de los pies y la cabeza. Para prevenir la mordedura señalaron el potencial uso de mosquiteros, mallas con las que puedan cubrir parte de sus casas, el humo producto de la quema de vegetación y la construcción de tarimas para dormir a fin de dificultar el acceso de estos animales.

[...] siempre hay ese techo donde se mete murciélago, para eso botamos (cogemos) cualquier planta y eso lo quemamos, ese humo los saca a todos. (Comunera). 
Sin embargo, los mismos informantes señalaron tener dificultades para implementar estos recursos, mencionado el elevado costo de los mosquiteros, inaccesibles para algunos, y el material precario de sus casas, explicando que el cubrirlas evita la ventilación y entrada de luz, originando excesivo calor (tabla 3).

Tabla 3. Principales acciones de prevención y control referidas por los entrevistados, según actores sociales

\begin{tabular}{|l|c|c|c|c|c|}
\hline \multicolumn{5}{|c|}{ Actores sociales. Comunidades Indígenas. Condorcanqui, Amazonas } \\
\hline $\begin{array}{l}\text { Acciones de prevención y } \\
\text { control manifestadas }\end{array}$ & Apus & Docentes & Pastor & Comuneros & Comuneras \\
\hline Mosquiteros & $\mathrm{X}$ & $\mathrm{X}$ & $\mathrm{X}$ & $\mathrm{X}$ & $\mathrm{x}$ \\
\hline $\begin{array}{l}\text { Tramperas para eliminar a los } \\
\text { murciélagos }\end{array}$ & $\mathrm{X}$ & $\mathrm{X}$ & & $\mathrm{X}$ & $\mathrm{x}$ \\
\hline $\begin{array}{l}\text { Charlas informativas personal } \\
\text { de salud }\end{array}$ & $\mathrm{X}$ & & & $\mathrm{X}$ & $\mathrm{x}$ \\
\hline Vacuna & $\mathrm{X}$ & & & $\mathrm{X}$ & $\mathrm{x}$ \\
\hline Cerrando las casas & $\mathrm{X}$ & & $\mathrm{X}$ & $\mathrm{X}$ & \\
\hline Vampirícelas & & & & $\mathrm{X}$ & $\mathrm{X}$ \\
\hline Infusiones y ungüentos & $\mathrm{X}$ & & & $\mathrm{X}$ & $\mathrm{x}$ \\
\hline
\end{tabular}

Fuente: elaboración propia

Un grupo menor afirmó que se podían desarrollar estrategias para el tratamiento de los efectos de la mordedura. Se mencionaron tres estrategias principales. Por un lado se señaló la bebida de infusiones de "piri-piri" (Cyperus spp.) por los participantes, un oxitócico que, desde su perspectiva, facilita la eliminación del agente causante de la enfermedad y que es usado tradicionalmente para evitar los efectos de la mordedura de serpientes. Una segunda estrategia consiste en la aplicación del extracto de Toé (Brugmansia suaveolens, árbol pequeño de la familia de las solanáceas) en la herida para curar al paciente matando al murciélago que regresa a morder nuevamente a su víctima, envenenándose al ingerir el Toé.

[...] le pones su sangre (extracto) de la planta lo mezclas y lo tomas, y aunque te muerda el murciélago ya no te va a dar rabia. (Comunero)

Se mencionó también que lavar la herida con limón, agua y/o jabón podía evitar el efecto de la mordedura. 
Aunque los entrevistados indicaban haber recibido información del personal de salud respecto a la vacuna contra la RS, buena parte de ellos desarrollaban diferentes discursos para oponerse o cuestionar la vacunación debido a aspectos religiosoculturales, socio-económicos y aspectos asociados a los servicios de salud (tabla 4) con una presencia variable de estos discursos entre una comunidad y otra.

Tabla 4. Cuestionamientos hacia la VAPEX contra la RS

\begin{tabular}{|c|c|c|c|c|c|c|}
\hline \multirow{2}{*}{$\begin{array}{l}\text { Causas de cuestionamiento a la } \\
\text { VAPEX contra la RS (frecuencia de } \\
\text { argumentos) }\end{array}$} & \multicolumn{6}{|c|}{ Comunidades participantes } \\
\hline & $\begin{array}{c}\text { CANAP } \\
1\end{array}$ & $\begin{array}{c}\text { CANAP } \\
2\end{array}$ & $\begin{array}{c}\text { CANAP } \\
3\end{array}$ & $\begin{array}{c}\text { CANAP } \\
4\end{array}$ & $\begin{array}{c}\text { CANAP } \\
5\end{array}$ & $\begin{array}{c}\text { CANAP } \\
6\end{array}$ \\
\hline \multicolumn{7}{|l|}{ Aspectos religiosos-culturales } \\
\hline Asociación con la marca del diablo & XXXX & $\mathrm{X}$ & $\mathrm{XX}$ & $\mathrm{X}$ & $\mathrm{X}$ & $\mathrm{X}$ \\
\hline $\begin{array}{l}\text { Las generaciones anteriores no } \\
\text { necesitaron de la vacunación para } \\
\text { subsistir }\end{array}$ & & $\mathrm{XX}$ & XXX & & $\mathrm{XX}$ & \\
\hline \multicolumn{7}{|c|}{ Aspectos relacionados con los servicios de salud } \\
\hline $\begin{array}{l}\text { Vacíos de información (vacuna sólo } \\
\text { para animales, sólo para niños, etc.) }\end{array}$ & $\mathrm{X}$ & $\mathrm{X}$ & & $\mathrm{XX}$ & XXX & \\
\hline $\begin{array}{l}\text { Los medicamentos provistos por el } \\
\text { estado son de baja calidad o están } \\
\text { vencidos }\end{array}$ & $\mathrm{X}$ & & & & $\mathrm{X}$ & $\mathrm{X}$ \\
\hline \multicolumn{7}{|l|}{ Aspectos socio-económicos } \\
\hline $\begin{array}{l}\text { Proyecto para exterminar al pueblo } \\
\text { Awajún y poseer su territorio para } \\
\text { fines económicos ocasionando } \\
\text { esterilidad o enfermedades, represalia } \\
\text { por conflictos sociales }\end{array}$ & $\mathrm{XX}$ & XXX & $\mathrm{XXX}$ & $\mathrm{XXX}$ & $\mathrm{XX}$ & $\mathrm{X}$ \\
\hline $\begin{array}{l}\text { Condicionalidad de la vacunación } \\
\text { para recibir ciertos servicios y } \\
\text { beneficios de salud como el SIS }\end{array}$ & & $\mathrm{X}$ & $\mathrm{X}$ & & & \\
\hline $\begin{array}{l}\text { Efectos negativos post aplicación: } \\
\text { Impidiendo temporalmente } \\
\text { desarrollar actividades laborales. } \\
\text { Afectando el crecimiento de los niños }\end{array}$ & $\mathrm{X}$ & $\mathrm{XXXX}$ & XXXX & & $\mathrm{X}$ & $\mathrm{X}$ \\
\hline
\end{tabular}

Fuente: elaboración propia 


\section{a. Aspectos religiosos - culturales}

Parte de los entrevistados, en Kayants y Shajain, consideraban que la vacunación no era necesaria por dos motivos principales. Por un lado se mencionó que, pese a haber recibido mordeduras de murciélagos a lo largo de su vida y especialmente cuando eran niños, no habían desarrollado la enfermedad. Este argumento se extendía, a sus antepasados. Varios de nuestros informantes mencionaron que ni sus padres ni abuelos enfermaron pese a no contar con la vacuna.

En segundo lugar, informantes de, por lo menos, tres de las seis comunidades estudiadas, indicaron que la rabia humana no existía y que se trataban de casos de daño o brujería, cuyo origen era sobrenatural y que debían prevenirse o tratarse utilizando recursos y terapeutas tradicionales.

En tercer lugar, los entrevistados mencionaron que el objetivo encubierto de la vacuna era "marcar" a la población Awajún con el "sello del diablo" o el "666." Según nuestros informantes ésta percepción surge como parte de un discurso de algunos grupos evangélicos que indican la existencia de un proyecto para marcar a aquellos que no son cristianos durante un periodo de persecución del cristianismo.

Anteriormente ella estaba de acuerdo con las vacunas, pero ahora hay comentarios de que la vacuna es malo, que trae enfermedades y que vienen con un veneno que con el tiempo te va a ir matando para que los indígenas se exterminen, demás por el código 666 , por eso es que ahora yo tengo miedo a las vacunas. Yo realmente creí que era efectiva pero con estos comentarios ya no me da confianza. (Comunero).

Hay religión bautista, nazareno, evangélico, etc. Hay hermanos pastores mismos que dicen que no pueden vacunarse porque dicen que con el tiempo va a salir el 666, que va a ver sello, eso es lo que comentan y entienden mal. (Apu).

\section{b. Aspectos socio-económicos}

Quienes perciben que la vacunación genera efectos negativos desarrollaron tres argumentos más frecuentes. El primero, encontrado en cuatro de las seis comunidades estudiadas, afirma que la vacuna tiene un efecto debilitante de carácter temporal que impedía el desarrollo normal de sus actividades laborales y cotidianas durante algunos días. Según sus testimonios, la vacuna genera dolor y malestar afectando, temporalmente, la capacidad del trabajo físico ya que genera malestares similares al reumatismo. Varios de nuestros entrevistados afirmaron que sus estrategias de sobrevivencia dependen directamente de alimentos que deben ser conseguidos, (recolectados, cazados o adquiridos) diariamente y que la 
pérdida de la capacidad de producción de uno de los miembros de sus unidades

domésticas podía afectar de manera importante su subsistencia, especialmente cuando se trataba de los jefes de familia.

Trabajo largo, permanente... ellos dedican a chacra y cuando hace esa inyección quedan débiles, no pueden hacer fuerza... ya malestar fiebre...hasta eso... hasta... como se llama este... granitos también sale dice... por la vacuna... como se llama... Alergia lo ataca. (Apu).

[...] yo entiendo que vienen diciendo para rabia pero cuando una vez que le ha vacunado empiezan a vacunarse y quedan débiles, sobre eso ya... yo de mi parte ya no lo acepte. (Apu).

En las comunidades de Kayants y Pajacusa se mencionó también que las vacunas afectaban negativamente el desarrollo de los niños, haciendo que perdieran peso, energía y sean más proclives a enfermarse. Este argumento, mencionado especialmente por madres de familia estaba acompañado de relatos de casos que ellas habían escuchado o atestiguado.

Porque sin vacuna me parece que yo estoy bien, sin vacuna, mis hijos están tranquilos contentos, y una vez me paso sobre que vacuna me han hecho y ya empezaron a enfermarse... entonces yo trato de evitarles, aunque estén desnutridos pero igual son, por lo tanto no puedo aceptar ningún tipo de vacunas. (Comunera).

La percepción más frecuentemente mencionada en la mayoría de las comunidades estudiadas indicaba que la vacunación tenía el objetivo encubierto de perjudicar al pueblo Awajún. Como parte de esta percepción se señalaron dos objetivos principales. El primero era "exterminar" al pueblo Awajun a través de la generación de enfermedades y/o esterilización de las personas vacunadas. Según esta percepción, existían dos motivos por las que el Estado actuaría en esa forma. Por un lado se mencionó que se buscaba beneficiar a las industrias extractivas interesadas en desarrollar proyectos en la zona, deshabitando la región. Por otro, se señaló que esta podía ser una represalia frente a las protestas desarrolladas por conflictos socio-económicos cuando solicitaron la derogación de dos decretos legislativos 1090 y 1064 que, desde su perspectiva, ponía en peligro la propiedad sobre su territorio.

Que es para que tengan vida corta, que es para que indígena que no aumente, por eso es que hay que vacunarse pa que tenga vida corta y luego se acabe el paisano y que puedan entrar para trabajar la mina libremente... todo eso está entrando esa idea... no sé si es cuando hablamos dicen mentira mentira a nosotros nos mienten... peor no sé de donde vendrá eso... yo tampoco puedo dar razones. (Comunero). 
Anteriormente ella estaba de acuerdo con las vacunas, pero ahora hay comentarios de que la vacuna es malo, que trae enfermedades y que vienen con un veneno que con el tiempote va a ir matando para que los indígenas se exterminen, demás por el código 666, por eso es que ahora yo tengo miedo a las vacunas. Yo realmente creí que era efectiva pero con estos comentarios ya no me da confianza. (Comunera).

Por ejemplo, de acuerdo con la versión vertida por autoridades de Ajachin, parte de su población consideraba que la vacunación tiene como objetivo la eliminación de la población Awajún. Desde su percepción, esta interpretación tenía que ver con el hecho que la campaña de vacunación contra la rabia se implementó en la zona en un contexto de protestas en memoria a conflictos socio-económicos. Entre la población corrió el rumor que la vacunación sería una represalia del Estado. Cabe mencionar que en la zona, durante el 2009 murieron 9 personas a casusa de un brote de RS y que en el 2010 se presentaron 04 casos de RS en la comunidad awajún de Uracusa, realizándose la vacunación post exposición con búsqueda de las personas mordidas.

[...] como ha habido un pequeño problema acá tanto en el paro de 5 de Junio eso, allí han sobreentendido que esa vacuna ha venido a eliminar a los, es decir al awajun, no? Bueno eso es un poco complicado, porque a través de la vacuna quieren que muera, con eso ha habido mal entendido. (Apu).

\section{c. Relacionados con los servicios de salud}

Un grupo de nuestros informantes pertenecientes a Ajachin y Shajain indicó que, pese a que la vacuna antirrábica podía ser efectiva, aquella que se utilizaba en la zona era de baja calidad o se encontraba vencida. Buena parte consideraba que, en general, los productos distribuidos gratuitamente por el Ministerio de Salud, especialmente aquellos que se destinaban a población indígena, solían ser "menos potentes" (tener menor efectividad) y estar en mal estado de conservación pudiendo ser dañinos para las personas. Entrevistados en ambas comunidades y de Seasme mencionaron también que la vacuna antirrábica distribuida estaba destinada a animales, por ejemplo a perros, y que no era apta para seres humanos. Un último argumento desarrollado por los entrevistados en la comunidad de Kayants, indica que las vacunas, en general, sirven para prevenir enfermedades en niños pero no en adultos.

A veces la gente piensa que les va a poner una ampolla pasada de su tiempo, eso les tiene miedo, piensan que el gobierno envía una medicina que no sirve y para no tener guardado lo envían para acá ese es el temor de las personas (Comunero). 
En Ajachin y Kayants se señaló también que los vacunados podían desarrollar enfermedades independientemente de la edad. Al respecto, se refirieron síndromes inespecíficos que no han sido atendidos de forma suficiente por el personal de salud como dolores al sistema musculo esqueleto, dolores de cabeza, problemas en el sistema digestivo, todos de tipo crónico y no como un efecto temporal.

\section{Escenario de incertidumbre}

Las personas entrevistadas para este estudio mencionaban más de un argumento a la vez y muchas indicaban no saber cuál de ellos era el más verás. Tampoco eran capaces de identificar con precisión las fuentes de estos argumentos, con excepción del discurso de la marca del diablo que asociaban a las iglesias evangélicas establecidas en la zona. Buena parte de nuestros informantes planteaba estos argumentos de tercera persona y de manera impersonal, señalando términos como "se dice..." o "han dicho" sin especificar las fuentes de información.

\section{Observación participante}

La zona de influencia del centro de salud (C.S) local de Uracusa abarca a las seis comunidades que participaron en este estudio, cuyo acceso requiere caminatas en algunos casos hasta por dos horas-, o utilizar medios de transporte terrestre o fluvial, generando costos económicos, los cuales no siempre pueden ser afrontados por los pobladores. Por otro lado, el C.S cuenta con un número limitado de personal salud y extensas áreas de jurisdicción, debido a esto no siempre es posible la realización de actividades extramurales, ya que implicaría la suspensión de las actividades rutinarias - preventivas y curativas- que se realizan en el C.S.

En relación a las visitas domiciliarias, se observó, que las viviendas permiten el ingreso de los murciélagos sea por ausencia de paredes, espacios en los techos u otras entradas y que existe poca utilización de mosquiteros. Durante las visitas domiciliarias realizadas, los trabajadores de salud tuvieron que dejar en ocasiones sus puestos con el propósito de alinearse a las actividades de las campañas generando retraso en las actividades rutinarias del C.S. Por otro lado algunos comuneros visitados optaban por salir de sus casas por el lado posterior a fin de evitar la vacuna en esa ocasión. El horario de estas visitas (principalmente matutinas) también resulto en ocasiones inapropiadas, ya que los comuneros se encontraban en sus chacras, piscigranjas u otras actividades, según reportaban 
los pobladores que atendieron al llamado. Finalmente, se observó que las autoridades locales tienen mayor énfasis en solicitar amplitud en la distribución de fármacos que en solicitar amplitud en la educación en Salud, priorizando la fase recuperativa-asistencial.

\section{Discusión}

Los hallazgos de este estudio pueden ser sintetizados brevemente en dos temas centrales: La negación o reconocimiento de la RS como una enfermedad mortal considerando ser causada por alguna infección o por poderes sobrenaturales, encontrándose percepciones variadas en relación al tratamiento y prevención a través de barreras físicas, las cuales dependen de factores socio-económicos relacionados a las condiciones en las que viven y en las que se desarrollan, mencionado con posterioridad a la vacunación. En relación a la vacunación contra la RS, los participantes mantienen variados argumentos que sostienen que la vacunación busca perjudicar de distintas maneras al desarrollo socio económico individual y colectivo de su pueblo, resaltando el clima de exclusión, pobreza, y de bajo acceso a servicios oportunos de salud en los que desarrollan.

A pesar de que el estudio no es generalizable sugiere una serie de pistas para mejorar las estrategias de abordaje de la prevención de la RS en CANAP. Muestra también, cómo el nivel de información que la población presenta sobre la RS y su prevención es difuso y complejo, escenario que puede crear un importante nivel de incertidumbre frente a una gran variedad de discursos desarrollados desde diferentes actores e instituciones (MORIN, 1994). Desde nuestra perspectiva la suspicacia que presenta buena parte de la población frente a la vacunación puede ser una respuesta ante la complejidad de la información que está circulando en la zona, pero también podría responder a la desconfianza y experiencia de exclusión frente al estado y los servicios de salud que llegan a estas comunidades.

La literatura reporta diversas evidencias relacionadas a la aceptabilidad sobre otros tipos de vacunas como la del papiloma virus humano (VAPVH) o el uso de quimio-profilácticos contra la malaria, que sostienen que la aceptación de este tipo de intervenciones preventivas puede ser baja cuando; la población percibe que la enfermedad es de bajo riesgo o que la persona no es susceptible a ella, o cuando se considera que la intervención puede significar una molestia o inconveniencia para el sujeto o para su entorno inmediato (MILES; HUBERMAN, 1994; TONG et 
al., 2007; MORIN, 1994). Estudios sobre barreras en la iniciación de esquemas de vacunación de varias dosis y estudios sobre creencias y conocimientos de sobre la VAPVH, concluyen que la aceptación de medidas preventivas se ve incrementada por un mayor conocimiento acerca de los síntomas, de la transmisión y de las formas de evitar la enfermedad (GUERRY et al., 2011; DO et al., 2009).

De manera similar a este reporte, otros estudios han documentado entre las principales barreras para la aceptación de una intervención de salud a la información difusa de; la enfermedad, de las causas que la origina y de las alternativas de prevención (entre ellas la vacunación), así como también a fuentes de información ambiguas como; la proveniente de creencias populares o de la influencia de pares. Sosteniendo que el conjunto de estos factores afecta en la decisión de optar por la vacunación (LAUREL; MILLS, 2013; MEPHAM et al., 2011). En relación a ello diversa literatura sostiene que existe una insistencia de explicar que las falla de las intervenciones en salud son causadas principalmente por factores culturales sean colectivos o individuales. Sin embargo, para ello sería necesario garantizar la facilidad del acceso a los servicios de salud a toda la comunidad vulnerable antes de atribuir falla en las intervenciones a raíz de los aspectos culturales del paciente (FARMER, 1997). Y por ejemplo, en estas comunidades son las facilidades al acceso las que principalmente no están totalmente garantizadas, resaltándose distintos factores (como aspectos, logísticos, de recursos humanos, de financiamiento) que debilitan la calidad de las intervenciones (CHAULET, 1987). Así, la pobreza y la inequidad son los grandes factores que influyen en que las personas no cumplan un tratamiento o en ese caso participen con pro-actividad de una campaña de salud como la vacunación (MARTENSEN, 2000). Esto no quiere decir que el conocimiento de la localidad sea irrelevante, sin embargo, este conocimiento suficiente o escaso no puede ser empleado para explicar la falla de las intervenciones sanitarias, principalmente cuando la calidad, acceso y cobertura de los servicios es insuficiente (KENDALL, 2000).

Resulta muy difícil explicar la causa de las enfermedades exclusivamente por cuestiones culturales individuales o colectivas dejando de lado las barreras estructurales para el acceso a los servicios de salud con calidad. El análisis cultural pierde sentido cuando no es insertado dentro del contexto socio-económico (MARTENSEN, 2000). Sosteniendo estos argumentos difundidos por Farmer (2001), estudios en Haití señalan que aun contando con atención gratuita y 
accesible al tratamiento antituberculoso, sus resultados fueron fuertemente relacionados con aspectos nutricionales y financieros y no con sus creencias sobre la causa de la enfermedad (MARTENSEN, 2000). Otros estudios sobre la propagación del VIH en Haiti, sostienen que en lugar de ser una consecuencia de prácticas culturales específicas, las condiciones socio-económicas de la comunidad dificultaron el cuidado adecuado de la salud y promoviendo la diseminación del virus (NACHMAN, 1993).

Las investigaciones de la medicina social muestra además de la diferencia entre la etiología de diversas enfermedades entre pacientes y proveedores de salud; el desacuerdo sobre las fallas de las intervenciones en salud (NACHMAN, 1993; CONRAD, 1985). En relación a ello podemos citar a un estudio realizado en un distrito mexicano en el cual las altas autoridades de salud atribuyeron a la pobreza, falta de educación, falta de motivación, creencias culturales y a la falta de comprensión sobre la importancia a la continuidad y adherencia al tratamiento de TB (RUBEL; GARRO, 1992). Un patrón similar fue encontrado en San Francisco donde los proveedores de salud asocian la falla del tratamiento a características culturales de los pacientes, sin embargo los pacientes mencionaron a barreras estructurales como la inconveniencia de los horarios u ubicación del establecimiento y rigidez en el orden de atención sin mayor flexibilidad a circunstancias especiales, como la dificultad de obtener citas para el mismo día o con el mismo médico para padres e hijos (SUMARTOJO, 1993). En tal sentido, enfatizando en estas enfermedades el rol de la cultural y no los roles de inequidad y pobreza, que son los puede ocasionar mayor daño, se sobrevalora la importancia de acciones individuales y se deja de lado la articulación de actividades sistemáticas efectivas de prevención en el fortalecimiento de la salud colectiva (MARTENSEN, 2000).

Las limitaciones en el acceso a los servicios de salud también influyen en las percepciones hacia sus servicios. El limitado contacto del personal de salud impide que se mantenga una comunicación continua con los pobladores, lo cual, a su vez, limita el número de actividades sanitarias extramurales. Esto fue reportado en una revisión sistemática sobre la prevención de la transmisión vertical del VIH, en la cual se encontró que uno de los principales factores que afectaron las intervenciones fue la distancia a los servicios y el número de visitas requerido (GOURLAY et al., 2013). Así también, en un estudio sobre la aceptación de otro tipo de vacuna, se 
encontró que los problemas con el transporte hacia los servicios de salud, impedían a las personas acudir a colocarse la vacuna (LAUREL; MILLS, 2013).

En el desarrollo de este estudio, el sesgo del idioma constituyó una potencial limitación. Para reducirla, se trabajó permanentemente con intérpretes locales capacitados en los objetivos y técnicas del estudio con el objeto de evitar perdida de la información obtenida en las entrevistas. Además, con el fin de evitar la sensación de confrontación durante las entrevistas, se emplearon entrevistas de poca estructuración, dando espacio a diálogos más abiertos e imparciales. Entre las fortalezas de este estudio se resalta la variabilidad de perspectivas recogidas desde distintos escenarios y actores sociales como autoridades locales, líderes religiosos, docentes, pobladores varones y mujeres.

Las percepciones recogidas hacia este tipo de vacunación manifiestan diversos cuestionamientos sobre su potencial beneficio o necesidad en la comunidad y están relacionadas a tanto a factores religiosos-culturales y socioeconómicos como a factores asociados al insuficiente acceso a los servicios de salud y a la fragmentada comunicación con el estado peruano.

Estos hallazgos representan oportunidades de abordaje en las estrategias preventivas promocionales donde, para hacer una real diferencia en estas intervenciones de salud, se debe explorar en detalle como la pobreza y la inequidad promueven la propagación de enfermedades como la RS evitando una sobrevaloración de los aspectos culturales mágico-religiosos (KENDALL, 2000). Así, focalizarnos en que finalmente las personas requieren de distintos esfuerzos, donde la vacunación representa uno de ellos, para prevenir o tratar sus enfermedades. Esto viabilizaría el reconocimiento de diversas barreras estructurales que impiden controlar alguna enfermedad y reflexionar por qué no han podido ser abordadas con éxito (BRIGGS, 2006; MENÉNDEZ, 1997). Al realizar el ejercicio de evaluar o reflexionar los caminos seguidos y las barreras estructurales con las que hemos tropezado antes podremos realizar un aporte cada vez más relevante para la salud colectiva (FARMER, 1997). ${ }^{1}$

\section{Agradecimientos}

Al Dr. Paul Pachas Chávez, por su compromiso en la orientación y coordinación de este estudio. Agradecemos también, a los pobladores, autoridades locales, las comunidades indígenas Awajún, como a las autoridades de salud, a la Dra. Ana María Navarro por su colaboración y aportes al presente estudio. 


\section{Referencias}

BASE DE DATOS DE PUEBLOS INDÍGENAS U ORIGINARIOS. Disponible en: <http://bdpi.cultura.gob.pe/>. Acceso: 17 diciembre 2014.

BERNARD, H.R. Social Research Methods: qualitative and quantitative approaches. Los Angeles: Sage Publications, 2012. 824 p.

BRIGGS, C.L. Perspectivas críticas de salud y hegemonía comunicativa: aperturas progresistas, enlaces letales. Rev Antropol Soc., n. 14, p. 101-24, 13 jan 2006.

BROWN, M.F. Una paz incierta : comunidades aguarunas frente al impacto de la Carretera Marginal, Lima, Perú : Centro Amazónico de Antropología y Aplicación Práctica, 1984.

CHAULET, P. Compliance with anti-tuberculosis chemotherapy in developing countries. Tubercle, suppl., p. 68, n. 19, 1987.

CONRAD, P. The meaning of medications: another look at compliance. Soc Sci Med., v. 20, n. 1, p. 29-37, 1985.

DePETRILLO, J. C. et al. Assessment of adherence to atovaquone-proguanil prophylaxis in travelers. J Travel Med., v. 17, n. 4, p. 217-20, Aug 2010.

DO, H. et al. HPV vaccine knowledge and beliefs among Cambodian American parents and community leaders. Asian Pac J Cancer Prev., v. 10, m. 3, p. 339-44, 2009.

FARMER, P. Social scientists and the new tuberculosis. Soc Sci Med., v. 44, n. 3, p. 347-58, Feb 1997.

FULLER, N. Relaciones de Género en la Sociedad Awajun. Lima: Care Perú, 2009. 100 p. Disponible en: <http://www.bvcooperacion.pe/biblioteca/bitstream/123456789/6415/1/ BVCI0006222.pdf>.

GÓMEZ J. Rabia. Protocolos de Vigilancia Epidemiologica. Parte I. Lima, Perú: Direccion General de Epidemiologia, 2004.

Nueva estrategia de intervención frente a la rabia transmitida por vampiros en comunidades indígenas amazónicas: "vacunación antirrábica de pre-exposición con vacunas de cultivo celular”. Boletín epideiológico, v. 20, n. 31, p. 647-9, 2011.

GOURLAY, A. et al. Barriers and facilitating factors to the uptake of antiretroviral drugs for prevention of mother-to-child transmission of HIV in sub-Saharan Africa: a systematic review. J Int AIDS Soc., n. 16, p. 185-88, 2013.

GUERRY, S.L. et al. Human papillomavirus vaccine initiation among adolescent girls in high-risk communities. Vaccine, v. 29, n. 12, p. 2235-41, Mar 9, 2011.

JOSHI, M.S.; LALVANI, A. "Home from home": risk perceptions, malaria and the use of chemoprophylaxis among UK South Asians. Ethn Health., v. 15, n. 4, p. 365-75, Aug 2010. 
KENDALL, C. Reviewed Work: Infections and Inequalities: The Modern Plagues by Paul Farmer. Med Anthropol Q., v. 14,. 3, p. 448-51, Sept 2000.

LAUREL, A.; MILLS, K.J.H. HPV vaccination among young adult women: a perspective from Appalachian Kentucky. Prev Chronic Dis., v. 10, p. E17, 2013.

MAINVILLE, J. R. Los awajún y wampís contra el Estado: una reflexion sobre antropología política. Investig Soc., v. 14, n. 24, p. 19-35, Apr 2014.

MARTENSEN, R.L. Book Review. N Engl J Med., v. 342, n. 18, p. 1374-5, May 2000.

MENÉNDEZ, E.L. El punto de vista del actor: homogeneidad, diferencia e historicidad. Relaciones, CIESAS, v. 69, p. 237-70, 1997.

MEPHAM, S. et al. Challenges in PMTCT antiretroviral adherence in northern KwaZuluNatal, South Africa. AIDS Care, v. 23, n. 6, p. 741-7, Jun 2011.

MILES, M.B.; HUBERMAN, A.M. Qualitative data analysis: an expanded sourcebook. Thousand Oaks: Sage Publications, 1994.

MINISTERIO DE SALUD. Casos de rabia transmitida por murciélagos y tasas de mordedura en la Dirección Regional de Salud de Amazonas. Lima: Ministerio de Salud, Dirección General de Epidemiologia, 2011a.

- Riesgo de transmisión de rabia silvestre ante la presencia de casos en el departamento

de Amazonas. Lima: Ministerio de Salud, Dirección General de Epidemiología, 2011b. Disponible en: <http://www.dge.gob.pe/portal/docs/alertas/2011/AE002.pdf>.

MORGAN, M.; FIGUEROA-MUÑOZ, J.I. Barriers to uptake and adherence with malaria prophylaxis by the African community in London, England: focus group study. Ethn Health, v. 10, n. 4, p. 355-72, Nov 2005.

MORIN, E. Introducción al pensamiento complejo. Barcelona: Gedisa, 1994.

NACHMAN, S.R. Wasted Lives: tuberculosis and other health risks of being Haitian in a U.S. Detention Camp. Med Anthropol Q., v. 7, n. 3, p. 227-59, Sept 1993.

NAVARRO, A.M.N. Ataques a humanos y transmisión de la rabia por murciélagos hematófagos en el Perú. En: SEMINARIO INTERNACIONAL MURCIÉLAGOS COMO TRANSMISORES DE RABIA. Programas e Resumos... p. 54-56; Sao Paulo-Brasil, 2001.

RYCHETNIK, L. et al. Criteria for evaluating evidence on public health interventions. $J$ Epidemiol Community Health, v. 56, n. 2, p. 119-27, Feb 2002.

SCRIMSHAW, C. M.; HURTADO, E. Rapid assessment procedures for nutrition and primary health care. Anthropological Approaches to Improving Programme Effectiveness. Los Angeles: UCLA, 1987.

RUBEL, A.J.; GARRO, L.C. Social and cultural factors in the successful control of tuberculosis. Public Health Rep., v. 107, n. 6, p. 626-36, 1992. 
SCHENSUL, S.L. Essential Ethnographic Methods: observations, interviews, and questionnaires. Altamira: Lanham, MD, 1999. 348 p.

SCHNEIDER, M.C. et al. Rabies transmitted by vampire bats to humans: an emerging zoonotic disease in Latin America? Rev Panam Salud Pública, v. 25, n. 3, p. 260-9, Mar 2009. SUMARTOJO, E. When tuberculosis treatment fails. A social behavioral account of patient adherence. Am Rev Respir Dis., v. 147, n. 5, p. 1311-20, May 1993.

TONG, A.; SAINSBURY, P.; CRAIG, J. Consolidated criteria for reporting qualitative research (COREQ): a 32-item checklist for interviews and focus groups. Int J Qual Health Care, v. 19, n. 6, p. 349-57, Dec 2007.

WORLD HEALTH ORGANIZATION. WHO Expert Consultation on Rabies: second report. Geneva: WHO, 2013. Available from: <http://apps.who.int//iris/ handle/10665/85346>. Accessed: Dec 17, 2014.

. The immunological basis for immunization series: module 17: rabies. Geneva: WHO, 2011.

. Rabies vaccines. WHO position paper, v. 82, n. 49-50, p. 425-436. Available from: <http://www.who.int/wer/2007/wer8249_50/en/>. Accessed: July 1 1 2015.

ZOONOSIS PM de SDG de S de las PD de AIC especial de. Norma técnica de salud para la prevención y control de rabia humana en el Perú: N.T.S. No. 052-MINSA/DGSP-V.01 [Internet]. 2008 [cited 2014 Dec 17]. Available from: http://bvs.minsa.gob.pe/local/ DGSP/770_DGSP242.pdf

\section{Nota}

${ }^{1}$ G. C Clavijo participó en la concepción del estudio, coordinaciones, ejecución, análisis de los datos y redacción del presente manuscrito. J. D. Arevalo participó en la ejecución del estudio, en la revisión crítica y relevante del contenido del manuscrito. V. L. O. Pretel participó en la ejecución del estudio, en la revisión crítica y relevante del contenido del manuscrito. J. P. Gutiérrez participó en el análisis de los datos y redacción del presente manuscrito. Todos los autores aprobaron la versión final del manuscrito. 
Percepçóes de vacinação contra a raiva em animais selvagens na população awajún $d a$ provincia de Condorcanqui, Amazonas, Peru, 2013

O objetivo do estudo foi determinar as perspectivas sobre a vacinação contra raiva silvestre na população awajún da província de Condorcanqui, Amazonas, em 2012. Foi realizada pesquisa qualitativa em seis comunidades por meio de entrevistas de baixa estrutura para a recolecção das perspectivas dos moradores, autoridades locais tradicionais e pessoal de saúde. As principais percepções recolhidas sobre as causas do RS foram atribuídas a: morcegos portadores de uma infecção, veneno ou portadores de algum poder sobrenatural; ou por efeito de feitiçaria. Foram expressos o efeito letal da enfermidade e diversas informaçōes sobre a possibilidade ou não de tratamento. $\mathrm{Na}$ prevenção da RS, mencionou-se principalmente uso de mosquiteiros, malhas, caçadores, chás e pomadas, depois as opções da vacina foram expressas. Em relação à vacinação, além de expressar a recepção de informações de saúde, foram dados argumentos para justificar sua rejeição ou questionamentos, como: o objetivo secreto de prejudicar ao desenvolvimento do povo Awajun; não ser necessária; e a geração de efeitos negativos em crianças e adultos. Estas perspectivas expressam diversos questionamentos enquanto seu potencial benéfico ou necessidade na comunidade e estão relacionadas tanto aos fatores socioeconômicos como as relaçôes de comunicação com o estado e o sistema de saúde peruano.

> Palavras-chave: vacinas antirrábicas; raiva; população indígena; percepção social. 


\section{Perceptions of wildlife rabies vaccination in Awajun population of the province of Condorcanqui, Amazonas, Peru, 2013}

The aim of this study was to determine the perspective for wild rabies (RS) vaccine in Awajún Peruvian population, Amazonas, 2012. A qualitative study was conducted in six communities across low structured interviews, designed to collect the perspective of the participants. We included community members, local authorities and health care personnel. Perception about RS causes was attributed to bats carrying infection, poison and supernatural harm, or the effect of witchcraft or damage. It was expressed the deadly effect and several information on the possibility or no treatment. To prevent the use of insecticide RS, tights, trappers, teas and ointments were mentioned, then came the vaccine. In relation to vaccination, besides expressing receipt of health information, were given arguments to oppose or question it as: the hidden objective to harm the Awajun people; not required by the population; and generation of adverse effects in children and adults. These perspectives addressing several questions about their potential benefit or right need it in the population and are associated to religious-cultural and socioeconomic factors as to the relationship with the health care systems and Peruvian government.

> Key words: wild rabies vaccine; indigenous population; perceptions. 